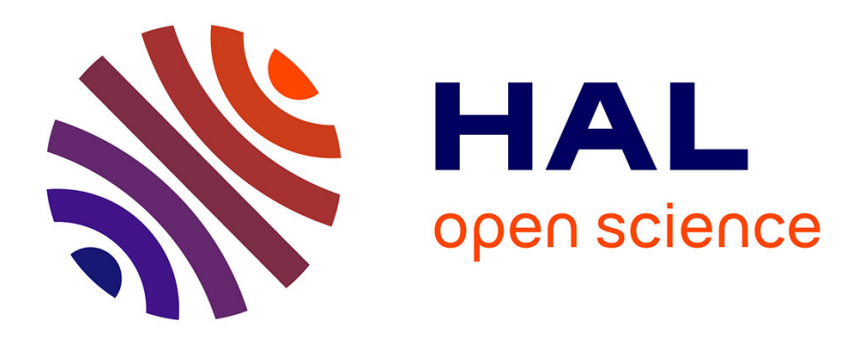

\title{
Modélisation basses fréquences d'un haut-parleur électrodynamique
}

\author{
Ph. Herzog, J. Le Roux
}

\section{To cite this version:}

Ph. Herzog, J. Le Roux. Modélisation basses fréquences d'un haut-parleur électrodynamique. Journal de Physique IV Proceedings, 1994, 04 (C5), pp.C5-225-C5-228. 10.1051/jp4:1994544 jpa-00253039

\section{HAL Id: jpa-00253039 https://hal.science/jpa-00253039}

Submitted on 1 Jan 1994

HAL is a multi-disciplinary open access archive for the deposit and dissemination of scientific research documents, whether they are published or not. The documents may come from teaching and research institutions in France or abroad, or from public or private research centers.
L'archive ouverte pluridisciplinaire HAL, est destinée au dépôt et à la diffusion de documents scientifiques de niveau recherche, publiés ou non, émanant des établissements d'enseignement et de recherche français ou étrangers, des laboratoires publics ou privés. 


\title{
Modélisation basses fréquences d'un haut-parleur électrodynamique
}

\author{
Ph. HERZOG et J.C. LE ROUX
}

Laboratoire d'Acoustique de l'Université du Maine, URA 1101 du CNRS, Avenue Olivier Messiaen, BP. 535, 72017 Le Mans cedex, France

\begin{abstract}
Although many methods are commonly available to get the Thiele-Small parameters of electrodynamic loudspeakers, the need to obtain a greater resolution and a good robustness led us to develop a method based on an analytical estimation of statistical values of these parameters, using a minimum number of measurements at arbitrary frequencies. The increased resolution obtained permitted us to point out some phenomena related to the heating of the suspension, and occuring during the aging of drivers. An iterative process applied to our method has also given a good estimate for another parameter related to the viscoelasticity of the suspension. This aspect is shown to be correlated to the material used for the cone surround, and may be responsible of some discrepancies observed betwen the parameter values obtained from different methods.
\end{abstract}

\section{INTRODUCTION}

Aux basses fréquences, le fonctionnement d'un haut-parleur est déterminé par un ensemble de six paramètres (paramètres de Thiele-Small [1]) dont quatre peuvent se déduire de la mesure de l'impédance électrique du transducteur. Plusieurs méthodes [1], [2], [3] permettent de déterminer ces paramètres de façon plus ou moins précise mais la résolution obtenue est difficilement quantifiable du fait de sensibilités variables aux limites du modèle, ou à des artéfacts de mesure. Nous avons de ce fait mis au point une méthode devant assurer une estimation relativement fiable de ces quatre paramètres, permettant ainsi de suivre avec une bonne résolution les évolutions de leurs valeurs.

\section{METHODE DE MESURE}

La méthode que nous avons développé nécessite un nombre minimum de trois mesures de l'impédance électrique du haut-parleur à des fréquences situées au voisinage de la résonance. L'estimation des paramètres se base sur deux relations caractéristiques de la forme analytique d'une résonance de type R-L-C: le lieu de l'impédance dans le plan complexe (cercle de Kennelly), et sa graduation fréquencielle. Une minimisation au sens des moindres carrés 
effectuée sur chacune de ces relations conduit à des expressions analytiques permettant de déduire les valeurs des paramètres à partir de l'ensemble des mesures, ce qui permet d'obtenir des valeurs statistiques de façon déterministe, et ainsi d'assurer une bonne répétabilité des estimations.

Une étude statistique et des calculs d'incertitude ont été développés pour évaluer les performances et optimiser l'emploi de cette méthode d'estimation. Il apparait qu'un bon compromis consiste à utiliser une dizaine d'acquisitions à des fréquences réparties juste autour de la résonance (gamme de fréquence correspondant à un demi cercle). En effet, l'augmentation du nombre de mesures accroit le temps d'acquisition sans donner une amélioration de la précision en rapport, tandis que l'utilisation d'une gamne de fréquence plus importante nuit à l'estimation de la fréquence de résonance. Ainsi, avec les appareils de mesure utilisés au laboratoire (intervalle d'incertitude de $1 \%$ sur l'amplitude et $0.01 \%$ sur la fréquence), la précision de la méthode d'estimation est de $2 \%$ sur le facteur de qualité mécanique, de $1 \%$ sur 1 a résistance équivalente aux pertes mécaniques et est meilleure que $0.1 \%$ sur 1 a fréquence de résonance; la résolution est de l'ordre de $10^{-4}$ sur cette dernière. Ces résultats sont obtenus à partir de mesures effectuées à un ensemble de fréquences arbitraires, parmi lesquelies ne sont conservées que celles qui vérifient les critères énnoncés ci-dessus; cette démarche permet ainsi d'automatiser intégralement le procédé et d'obtenir une estimation très rapide.

\section{RESUITATS}

Nous présentons quelques résultats issus de l'application de notre méthode d'estimation a la mesure des haut-parleurs dans leur gamme de fonctionnement linéaire; nous avons entre autres visualisé l'évolution au cours du rodage des caractéristiques d'un haut-parleur. Les figures 1 et 2 montrent les fluctuations de la compliance et de la résistance correspondant aux pertes mécaniques. La résolution obtenue pour les estimations permet d'observer un phénomène de "chauffage" des suspensions qui survient après chaque interruption de l'alimentation du haut-parleur (figure 1).

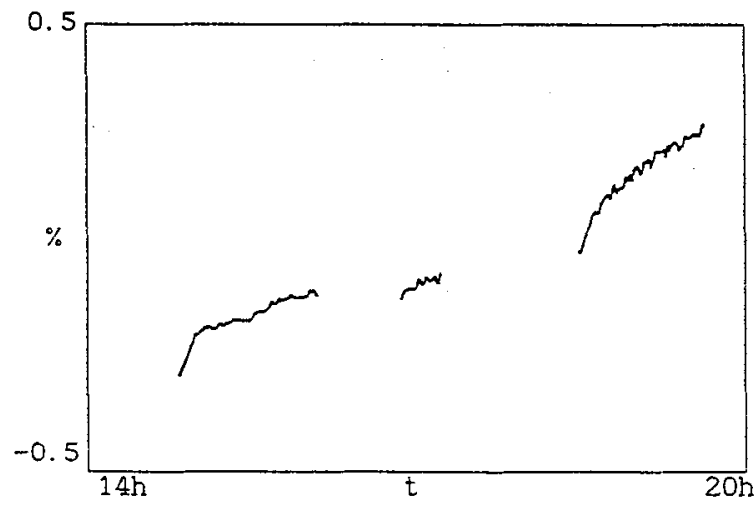

Figure 1

Evolution de la compliance d'un haut-parleur au cours du rodage. 


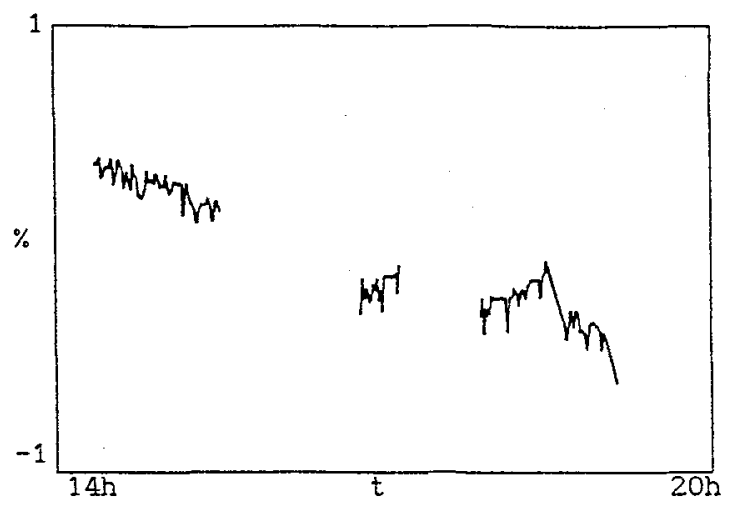

Figure 2

Evolution de la résistance correspondant aux pertes mécaniques a'un haut-parleur.

Même à faible niveau d'excitation, il apparait cependant des écarts entre le modèle linéaire utilisé et le comportement réel de certains haut-parleurs, en particulier du fait que la viscoélasticité des suspensions n'est pas prise en compte dans ia modélisation (figure 3). Cette particularité des suspensions, présente sur bon nombre de haut-parleurs, est souvent masquée par une évaluation erronée de la partie imaginaire de I'impédance électrique du moteur aux basses fréquences. Cette dernière est assimilée à une inductance pure dont la valeur (mesurée vers $1 \mathrm{kHz}$ ) est très faible; son influence est alors supposée négligeable aux basses fréquences. En réalité, l'existence même à ces fréquences d'une partie imaginaire significative pour l'impédance électrique du moteur compense en apparence l'effet de la viscoélasticité des suspensions sur le tracé de la courbe d'impédance, faussant de ce fait l'estimation des paramètres du haut-parleur.

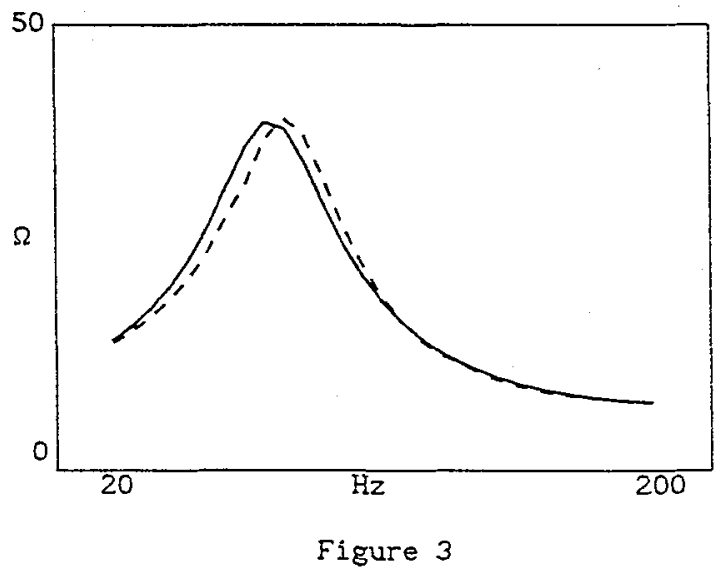

Effet de la viscoélasticité des suspensions sur le module de l'impédance électrique - - impédance mesurée

- impédance théorique. 
L'introduction de deux processus itératifs dans la méthode d'estimation présentée précédemment, et l'emploi d'une modélisation empirique pour évaluer l'impédance électrique du moteur aux basses fréquences [4], autorisent l'évaluation avec une précision suffisante d'un paramètre supplémentaire (B) traduisant la viscoélasticité des suspensions [5]. L'application de ce procédé à plusieurs modèles de haut-parleurs permet de dégager certaines tendances en fonction de la nature du matèriau de la suspension extérieure (figure 4). On constate notamment que les suspensions "caoutchouc" sont les plus viscoélastiques et qu'en aucun cas le modèle de Small ( $B=0$ ) n'est vérifjé.

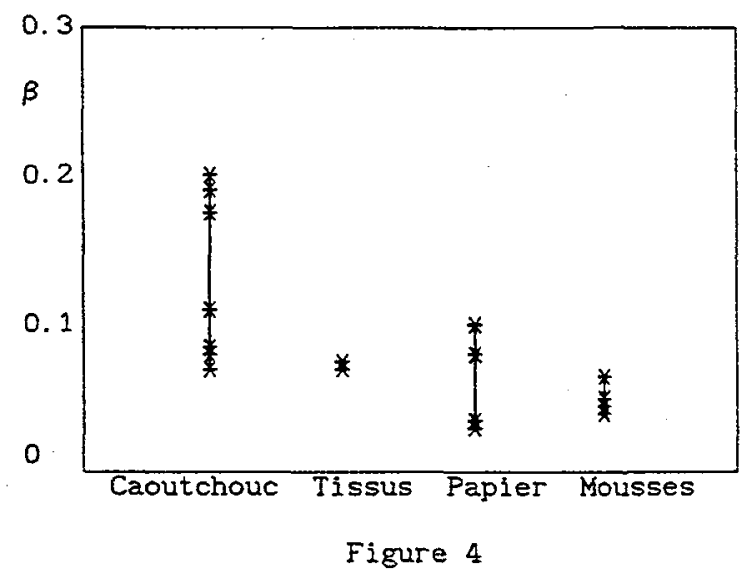

Effet du materiau de la suspension extérieure sur la viscoélasticité de la compliance totale.

\section{REFERENCES}

[1] R.H. SMALI, "Direct-radiator loudspeaker system analysis", J. Audio Eng. Soc., Vol 20 , June 1972.

[2] J.J.M.KREUTZ \& J.PANZER, "Determination of the quality factor of loudspeakers by a statistical method", $90 \mathrm{th}$ Convention of the Audio Engineering Society, Paris, February 1990 , preprint $n^{\circ} 3039$.

[3] W.MARSHALI LEACH JI., R.W.SCHAFER, T.P.BARWWELI, "Time domain measurement of loudspeaker driver parameters", I.E.E.E. Trans. Acoust. Speech. Signal Proc., Vol ASSP-27, December 1979.

[4] J.R.WRIGHT, "An empirical model for loudspeaker motor impedance", J.Audio Eng. Soc., Vol. 38, October 1990.

15] M.H. KUUDSEN, P. HANSEN, J.G. JENSEN, "The significance of viscoelastic effects in loudspeaker parameter measurements", 88th Convention of the Audio Engineering Society, Montreux, March 1990, preprint n²905. 